\title{
Asteroseismology in Praesepe: the case of EP Cnc
}

\author{
J.A. Belmonte, A. Claret and F. Pérez Hernández \\ Instituto de Astrofísica de Canarias, 38200 La Laguna, Tenerife, Spain
}

\begin{abstract}
On the 1992 STEPHI IV campaign, the multi-periodic $\delta$ Scuti stars BN Cnc and BU Cnc of the Praesepe cluster were monitored during a three-week, three-continental run (Belmonte et al. 1994). Five and six frequency peaks, respectively, were recorded as a result of the campaign. In a subsequent paper (Pérez Hernández et al. 1995, hereafter Paper I), an attempt to identify these frequency peaks as pulsation modes was done. An important point is that both stars belong to the same cluster and, hence, more severe constraints than for isolated stars can be imposed. Recently, Breger et al. (1994) have reported results on EP Cnc, another $\delta$ Scuti in Praesepe. This star is also multi-periodic, showing three frequency peaks above the noise level. In the present work we extend our analysis to this star.
\end{abstract}

\section{Discussion}

As a matter of fact, EP Cnc is more luminous than BN and BU Cnc and presumably all the hydrogen in its convective core has been depleted, as opposite to BN Cnc and BU Cnc. In Paper I, the model parameters were chosen in order to fit the observed frequencies of BN Cnc and BU Cnc. The range of ages considered were also obtained from the theoretical analysis of the observed frequencies. Although for EP Cnc (as well as for BN Cnc and BU Cnc), other models can also fit the observed frequencies, to be consistent, we shall assume the same parameters for the three stars. The models corresponding to each star are constrained by the observed magnitudes and effective temperatures. In this sense, a mass between $2.3 M_{\odot}$ and $2.4 M_{\odot}$ can be estimated for EP Cnc.

Frequencies are computed in the linear and adiabatic approximation. Rotation effects are considered up to second order, namely

$$
\nu_{n l m}-\nu_{n l}^{(0)}=-m\left(1-C_{n l}\right) \nu_{r o t}+\left(X_{n l}+m^{2} Y_{n l} \frac{\nu_{r o t}^{2}}{\nu_{n l}^{(0)}}\right.
$$

where $\nu_{n l}^{(0)}$ is the unperturbed frequency and $\nu_{\text {rot }}$ the rotation rate of the star.

As for BN and BU Cnc, we start the identification of the frequency peaks by looking for possible radial modes. This can be done by comparing the observed period ratios with the theoretical ones. For EP Cnc, evolutionary stages before and after the red hook have been considered. In contrast to BN and BU Cnc, for which two radial modes $(n=5$ and $n=6)$ can be present, for EP Cnc only one of the frequency peaks can be a radial model, either $n=2$ or $n=3$. The 
fundamental mode $n=1$ is unlikely. It is interesting to note that this latter fact is due to the inclusion of the frequency shift caused by rotation and not to the restrictive set of stellar models considered here.

There are at least two non-radial modes. When considering all the theoretical modes with $l=1$ and $l=2$, it is found that tens of them are present in the observed frequency range. However, not all of them are of the same nature. The $l=2$ modes can be regarded as $g$-modes, while for the $l=1$ modes there are both $g$-modes and modes which are trapped predominantly in the envelope. Envelope-trapped modes have more relative energy in the upper layers than $g$ modes and rotational splitting parameters closer to those of $p$-modes. We note that this situation is typical for these kind of stars (see e.g. Dziembowski \& Goode 1992 for the case of $\theta^{2}$ Tau).

In the observed frequency range, and for our set of models, there is just one $(n, l=1)$ envelope-trapped mode. In principle it can be thought that this $p$-like mode is the more likely to be detected since, for a given energy, it has the largest surface amplitude. However the (unknown) excitation mechanism could give a substantially larger energy to some of the $g$-modes.

On the other hand, the rotational frequency splittings of the $\mathrm{g}$ and envelopetrapped modes are rather different. If there were no $g$-modes, then we found that $\nu_{2}=67.0 \mu \mathrm{Hz}$ is a radial mode with $n=2$ and $\nu_{1}=63.3 \mu \mathrm{Hz}$ and $\nu_{3}=75.4 \mu \mathrm{Hz}$ correspond to an $l=1$ envelope-trapped mode split by rotation. The difference between $\nu_{3}$ and $\nu_{1}$ is $12.1 \mu \mathrm{Hz}$, while from the observed value of $V \sin i$ and our models, it follows that $\nu_{\text {rot }}>7.2 \mu \mathrm{Hz}$. If they correspond to $m= \pm 1$, then $C_{n l}>0.16$. This is compatible with an envelope-trapped mode.

It is interesting to consider a frequency peak at $72.9 \mu \mathrm{Hz}$, although, following Breger et al. (1994), it is below the noise level. If $g$-modes are not present in the spectrum then it must correspond to the $m=0$. From eq. (1) the value of $Y_{n l}$ can be estimated, yielding $Y \sim-4$, which is in the range of values expected for envelope-trapped modes. Hence, even in this case, the presence of $g$-modes is not necessary.

\section{References}

Belmonte, J.A., Michel, E., Alvarez, M., Jiang, S.Y., Chevreton, M., Auvergne, M., Liu, Y.Y., Goupil, M.J., Baglin, A., Roca Cortés, T., Mangeney, A., Dolez, N., Valtier, J.C., Massacrier, G., Sareyan, J.P., Schmider, F.X., \& Vidal I. 1994, A\&A, 283, 121

Breger, M., Martin, B., Garrido, R., Jiang, S.Y., Li, Z.P., Hube, D.P., Stich, J., Ostermannn, W., \& Paparo M. 1994, A\&A, 281, 90

Pérez Hernández, F., Claret, A., \& Belmonte, J.A. 1995, A\&A, in press

Dziembowski, W.A., \& Goode, P.R. 1992, ApJ, 394, 670 